九州大学学術情報リポジトリ

Kyushu University Institutional Repository

\title{
APPROXIMATE EXTENSIONS IN DEFAULT LOGIC
}

Shi, Yi-Hua

Department of Information Systems, Interdisciplinary Graduate School of Engineering Sciences, Kyushu University

Arikawa, Setsuo

Research Institute of Fundamental Information Science, Kyushu University

https://doi.org/10.5109/3153

出版情報 : Bulletin of informatics and cybernetics. 25 (1/2), pp.7-19, 1991-09. Research Association of Statistical Sciences

バージョン :

権利関係: 


\title{
RIFIS Technical Report
}

Sub-extensions and Approximate Extensions

in Reiter's Default Logic

\author{
Yi-Hua Shi \\ Setsuo Arikawa
}

September, 1991

Research Institute of Fundamental Information Science

Kyushu University 33

Fukuoka 812, Japan

E-mail: shi@rifis1.sci.kyushu-u.ac.jp Phone: 092(641)1101 Ext.4484 


\title{
Sub-extensions and Approximate Extensions in Reiter's Default Logic
}

\author{
Yihua Shi and Setsuo Arikawa \\ Department of Information Systems, Kyushu University 39, \\ Kasuga, Fukuoka 816, Japan \\ E-mail: shi@rifis.sci.kyushu-u.ac.jp
}

\begin{abstract}
The extension, one of the most important notions in Reiter's default logic, is not computable in general. This paper proposes new notions of sub-extension and approximate extension which can successively be constructed from a given default theory. By introducing a time-bound into them, we show that every step of our construction terminates in a finite time. We also prove that the set of approximate extensions contains the set of extensions in Reiter's sense.
\end{abstract}

\section{Introduction}

In order to apply AI systems in more unrestricted domains, more efficient and flexible methods for representing and inferring their knowledge are required. Reiter's default theory [3] gives a theoretical foundation to meet such a requirement. His default reasoning can informally be described as the process of jumping to conclusions based on what is normally the case. An extension, an important notion to describe default theories, is a maximal set of results reasoned from a default theory.

The extension, however, can not successively be constructed, because it is defined by using itself in applying the defaults. To solve this problem, Yuasa and Arikawa([9]) introduced the notion of pseudo extension which can successively be constructed from a given default theory. Since the termination of derivations is not generally guaranteed in first order logic, the pseudo extensions are still not computable. In order to effectively construct the extensions, we need to restrict the time allowed for every construction step.

In this paper, we pay attention to the termination of construction steps. Based on our time-bounded reasoning $[5,7]$, we propose the notions of approximate extensions and finite sub-extensions, and show that such extensions can be successively constructed and every construction step terminates in a finite time. We also prove every Reiter's extension is an approximate extension.

In Section 3, we introduce the time-bound into the construction of extensions, and discuss the reliability of knowledge in extensions and the influence of time-bound on the 
correctness of reasoned results. In Section 4, we give the definitions of the $\mathrm{i}$-th finite sub-extensions and approximate extensions which can successively be constructed and terminate every construct step in a finite time, and show that every extension is an approximate extension. Section 5 illustrates the construction on the sub-extensions and approximate extensions by some examples. Section 6 gives the concluding remarks of this paper.

\section{Preliminaries}

\subsection{Default reasoning and extensions}

Let $L$ be a first order language. Well-formed formulas (wff for short) are formed in the usual way. A wff is closed if it does not contain any free variable. Then a default rule(or default) is a rule of the form

$$
\frac{\alpha: M \beta_{1}, \ldots, M \beta_{m}}{\omega},
$$

where $\alpha, \beta_{1}, \ldots, \beta_{m}, \omega$ are wffs in $L$, We call $\alpha$ a prerequisite, $\beta_{1}, \ldots, \beta_{m}$ justifications, and $\omega$ a consequence. $M$ is a meta-operator which can be read as "it is consistent to assume". The default is closed if $\alpha, \beta_{1}, \ldots, \beta_{m}, \omega$ are closed wffs.

Let $D$ be a set of defaults, and $W$ be a set of closed wffs. Then $\Delta=(D, W)$ is called a default theory. A default theory $\Delta$ is closed if every default in $D$ is closed.

For a set $D$ of default rules, we define $\operatorname{Cons}(D)$, the set of all consequences of $D$, by

$$
\operatorname{Cons}(D)=\left\{\begin{array}{l|l}
\omega & \frac{\alpha: M \beta_{1}, \ldots, M \beta_{m}}{\omega} \in D
\end{array}\right\}
$$

For a set $S$ of closed wffs and a closed wff $\omega$, we use $S \vdash \omega$ to denote that $\omega$ is derived from $S$, and $S \forall \omega$ to denote that $\omega$ is not derived from $S$. We also define the set $T h(S)$ by

$$
T h(S)=\{\omega \mid \omega \text { is a closed wff and } S \vdash \omega\}
$$

Definition 2.1 (Reiter [3]) Let $\Delta=(D, W)$ be a closed default theory, $S \subseteq L$ be a set of closed wffs, and $\Gamma(S)$ be the smallest set satisfying the following three conditions:

(1) $W \subseteq \Gamma(S)$,

(2) $T h(\Gamma(S))=\Gamma(S)$,

(3) If $\frac{\alpha: M \beta_{1}, \ldots, M \beta_{m}}{\omega} \in D, \alpha \in \Gamma(S), \neg \beta_{1}, \ldots, \neg \beta_{m} \notin S$ then $\omega \in \Gamma(S)$.

$A$ set $E \subseteq L$ satisfying $\Gamma(E)=E$ is an extension of $\Delta$.

An intuitive characterization of the extensions is given by the following theorem: 
Theorem 2.1 (Reiter [3]) Let $\Delta=(D, W)$ be a closed default theory, $E \subseteq L$ be a set of closed $w f f s$, and $E_{i}(i \geq 0)$ be sets of closed wffs defined as follows:

$$
\begin{aligned}
& E_{0}=W \\
& E_{i+1}=T h\left(E_{i}\right) \cup\left\{\begin{array}{l|l}
\omega & \begin{array}{l}
\alpha: M \beta_{1}, \ldots, M \beta_{m} \\
E_{i} \vdash \alpha \\
E \nvdash \neg \beta_{1}, \ldots, \neg \beta_{m}
\end{array}
\end{array}\right\}
\end{aligned}
$$

Then $E$ is an extension of $\Delta$ if and only if

$$
E=\bigcup_{i=0}^{\infty} E_{i}
$$

We can use the above theorem to check whether a given set of closed wffs is an extension or not, but since Reiter used $E$ in the definition of itself, we can not use the theorem to construct extensions from a given closed default theory.

\section{$2.2 \quad$ Pseudo extensions}

In order to avoid the self-recursive definition in Theorem 2.1, Yuasa and Arikawa introduced a new kind of extensions called pseudo extensions.

Definition 2.2 (Yuasa and Arikawa [9]) Let $\Delta=(D, W)$ be a closed default theory and $F_{i}(i \geq 0)$ be sets of closed wffs defined as follows:

$$
\begin{aligned}
F_{0} & =W \\
F_{i+1} & =\operatorname{Th}\left(F_{i}\right) \cup \operatorname{Cons}\left(D_{F_{i}}\right) \quad(i \geq 0),
\end{aligned}
$$

where $D_{F_{i}}$ is a subset of

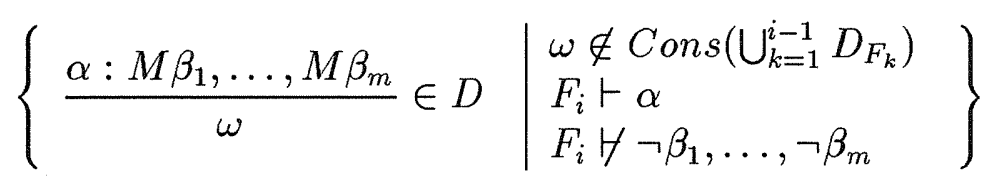

such that $D_{F_{i}} \neq \phi$ if the above set is not empty. Then we call

$$
F=\bigcup_{i=0}^{\infty} F_{i}
$$

a pseudo extension of the theory $\Delta$.

By using $F_{i}$ instead of $E$ at checking $M \beta_{j}$, the pseudo extensions can successively be constructed from a given closed default theory. There is the following relation between Reiter's extensions and pseudo extensions, which shows that every extension is a pseudo extension.

Theorem 2.2 (Yuasa and Arikawa [9]) Let $\mathbf{E}$ be the set of all extensions of a closed default theory $\Delta$, and $\mathbf{F}$ be the set of all pseudo extensions of $\Delta$. Then $\mathbf{E} \subseteq \mathbf{F}$. 


\section{Reliability and correctness}

Although the pseudo extensions can successively be constructed, there still remain two problems:

(a) We can not know how long it takes to compute an $F_{i}$ from $F_{i-1}$, since the termination of computation of $T h, F_{i} \vdash \alpha$, and $F_{i} \forall \neg \beta$ in first order logic are generally not guaranteed.

(b) We can not know when the sequence of $F_{1}, F_{2}, \ldots$ converges.

The problem (a) should be more serious than (b) when we successively construct some extensions or parts of them.

In order to solve the first problem (a), we introduce the time-bounded reasoning [5, 7] into the computation of extensions. That is, we use "ト $n$ " instead of "ト".

Let $\alpha$ be a closed wff, $S, A_{1}, A_{2}, \cdots, A_{k}$ be sets of closed wffs. We define the following notations:

(1) $S \vdash_{n} \alpha: \alpha$ is derived from $S$ in time $n$.

(2) $S \nvdash_{n} \alpha$ : $\alpha$ cannot be derived from $S$ in time $n$.

(3) $S \nvdash_{n}^{F} \alpha$ : all derivations of $\alpha$ from $S$ are fail in time $n$.

(4) $S \nvdash_{n}^{I} \alpha$ : whether or not $\alpha$ is derived from $S$ cannot be decided in time $n$.

(5) $T h_{n}(S)=\left\{\alpha \mid \alpha\right.$ is a closed wff and $\left.S \vdash_{n} \alpha\right\}$

(6) $T h_{n}^{\prime}\left(A_{1} \cup A_{2} \cup \cdots \cup A_{k}\right)=T h_{n}\left(A_{1} \cup A_{2} \cup \cdots \cup A_{k}\right)-T h_{n}\left(A_{1} \cup \cdots \cup A_{k-1}\right)$

Then, clearly

$$
S \nvdash_{n} \alpha \Leftrightarrow\left(S \nvdash_{n}^{F} \alpha \text { or } S \nvdash_{n}^{I} \alpha\right),
$$

\subsection{Default theories and reliability}

A default theory $\Delta=(D, W)$ consists of a complete knowledge part $W$ (such as a first order theory) and an incomplete knowledge part $D$ (such as a commonsense knowledge). They are different in reliability. Generally, the closed wffs in an extension $E$ can be divided into knowledge and beliefs. A closed wff is knowledge of theory $\Delta$ if it belongs to every extension of theory $\Delta$. A closed wff is a belief of theory $\Delta$ if it belongs to some extensions of theory $\Delta$. Obviously, the closed wffs in $T h(W)$ are knowledge, and every knowledge is a belief.

Theorem 2.1 and Definition 2.2 do not distinguish the results inferred only from $W$ from those from both $D$ and $W$. When we pay attention only to one extension, such a distinction is not important. However, when we consider the set of extensions, or introduce the time-bound to the computation of extensions, the classification of them becomes very 
important. An extension may be taken as a possible belief set of the agent. If $\alpha \in T h(W)$, then $\alpha$ is true in every possible belief set. But $\alpha \in E$ just means that $\alpha$ is true in the possible belief set $E$.

Furthermore, because the defaults, i.e., commonsense knowledge, are incomplete, the use of defaults may cause some invalid results $[4,1]$; that is to say, there may be some beliefs in some extensions that do not match with our intuition.

Thus, it is necessary to distinguish the results inferred only from $W$ from those inferred from both $D$ and $W$.

\subsection{Time-bound and correctness}

In order to make every step of successive construction finish in a finite time, we introduce a time-bound $n$ into the computation of extensions. Thus, we need to interpret $M \beta_{j}$ in defaults as $S E_{i-1} \nvdash_{n}^{F} \neg \beta_{j}$ or $S E_{i-1} \nvdash_{n}^{I} \neg \beta_{j}$, where $S E_{i-1}$ is the $(i-1)$-st finite subextension; that is, we weaken the justifications of defaults as follows

$$
\underbrace{\left(M \beta_{j}=E \nvdash \neg \beta_{j}\right)}_{\text {Reiter's }} \geq \underbrace{\left(F_{i} \not \forall \neg \beta_{j}\right)}_{\text {Yuasa's }} \geq \underbrace{\left(S E_{i-1} \nvdash_{n}^{F} \neg \beta_{j}\right) \geq\left(S E_{i-1} \nvdash_{n}^{I} \neg \beta_{j}\right)}_{\text {ours }} .
$$

To a default rule

$$
\frac{\alpha: M \beta_{1}, \cdots, M \beta_{m}}{\omega},
$$

we can take $M \beta_{1}, \ldots, M \beta_{m}$ as the justifications of $\alpha$ implying $\omega$. Then, weakening the justifications leads to decrease the level of correctness of the consequences; that is, $\alpha$ and $\omega$ are different in the level of correctness. Thus, we need to classify them.

\section{Sub-extensions and approximate extensions}

Now we define the notion of the " $i$-th finite sub-extension" as follows.

Definition 4.1 Let $\Delta=(D, W)$ be a closed default theory, the time-bound be $n$. Then we divide the $i$-th finite sub-extension $S E_{i}$, a set of closed wffs, into three parts:

$$
S E_{i}=K_{i} \cup B_{i} \cup G_{i} \quad(i \geq 0)
$$

which are constructed as follows:

$$
\begin{aligned}
& K_{0}=W \\
& B_{0}=G_{0}=\phi \\
& K_{i+1}=T h_{n}^{\prime}\left(K_{i}\right), \\
& B_{i+1}=T h_{n}^{\prime}\left(K_{i} \cup B_{i}\right) \cup \operatorname{Cons}\left(D_{i}^{\prime}\right) \\
& G_{i+1}=T h_{n}^{\prime}\left(K_{i} \cup B_{i} \cup G_{i}\right) \cup \operatorname{Cons}\left(D_{i}^{\prime \prime}\right)
\end{aligned}
$$


where $D_{i}^{\prime}$ is an empty set or a singleton set $\{\delta\}$ such that

$$
\begin{aligned}
& \delta=\frac{\alpha: M \beta_{1}, \ldots, M \beta_{m}}{\omega} \in D, \\
& \omega \notin K_{i+1}, \quad K_{i}, B_{i} \vdash_{n} \alpha, \quad \text { and } \\
& K_{i}, B_{i} \nvdash_{n}^{F} \neg \beta_{1}, \ldots, K_{i}, B_{i} \nvdash_{n}^{F} \neg \beta_{m},
\end{aligned}
$$

and $D_{i}^{\prime \prime}$ is an empty set or a singleton set $\{\delta\}$ such that

$$
\begin{aligned}
& \delta=\frac{\alpha: M \beta_{1}, \ldots, M \beta_{m}}{\omega} \in D, \\
& \omega \notin K_{i+1} \cup B_{i+1}, \quad K_{i}, B_{i}, G_{i} \vdash_{n} \alpha, \quad \text { and } \\
& K_{i}, B_{i}, G_{i} \nvdash_{n} \neg \beta_{i}, \quad(j=1, \ldots, m) .
\end{aligned}
$$

$K_{i}, B_{i}$, and $G_{i}$ are called the knowledge part, the belief part, and the guess part of $S E_{i}$, respectively.

Proposition 4.1 Let $S E_{i}=K_{i} \cup B_{i} \cup G_{i}(i=1,2, \cdots)$ be a sequence of sub-extensions constructed by Definitions 4.1. Then,

$$
\begin{aligned}
& K_{1} \subseteq K_{2} \subseteq \cdots \subseteq K_{j} \subseteq \cdots \\
& B_{1} \subseteq B_{2} \subseteq \cdots \subseteq B_{j} \subseteq \cdots \\
& G_{1} \subseteq G_{2} \subseteq \cdots \subseteq G_{j} \subseteq \cdots
\end{aligned}
$$

Definition 4.2 Let $\Delta=(D, W)$ be a closed default theory, $S E_{i}=K_{i} \cup B_{i} \cup G_{i} \quad(i=$ $1,2, \cdots)$ be any sequence of finite sub-extensions constructed by Definitions 4.1. Then, $A E=K \cup B \cup G$, an approximate extension of the theory $\Delta$, is a set of closed wffs, where

$$
K=\bigcup_{i=0}^{\infty} K_{i}, \quad B=\bigcup_{i=0}^{\infty} B_{i}, \quad G=\bigcup_{i=0}^{\infty} G_{i} .
$$

The wffs in $K$ is called knowledge, whose reliability is the highest. The wffs in $B$ is called belief, whose reliability is medium. The wffs in $G$ is called guess, whose reliability is the lowest. By Definition 4.1 and Definition 4.2, there exists a unique fixpoint $K$ for $K_{i}(i=1,2, \cdots)$, but there may be many fixpoints for $B_{i}$ and $G_{i}(i=1,2, \cdots)$ by the selection of $D_{j}^{\prime}$ and $D_{j}^{\prime \prime}$. Obviously, $K=\bigcup_{i=0}^{\infty} K_{i}=T h(W)$ is a minimal approximate extension which ignores the part of defaults.

Theorem 4.2 Let $\Delta=(D, W)$ be a closed default theory, $E \subseteq L$ be a set of closed wffs, and $E_{i}^{\prime}(i \geq 0)$ be sets of closed wffs defined as follows:

$$
\begin{aligned}
E_{0}^{\prime} & =W, \\
E_{i}^{\prime} & =T h\left(E_{i-1}^{\prime}\right) \cup \operatorname{Cons}\left(\left\{\delta_{i}\right\}\right),
\end{aligned}
$$

where

$$
\delta_{i}=\frac{\alpha: M \beta_{1}, \ldots, M \beta_{m}}{\omega} \in D
$$


is a default which satisfies $E_{i-1}^{\prime} \vdash \alpha, E \nvdash \neg \beta_{1}, \ldots, \neg \beta_{m}$. Then, $E$ is an extension of $\Delta$ if and only if

$$
E=\bigcup_{i=0}^{\infty} E_{i}^{\prime}
$$

Proof. Trivial from the definitions.

Theorem 4.3 Let $E$ be any extension of closed default theory $\Delta$. Then, there exists an approximate extension $A E$ of $\triangle$ such that $A E=E$.

Proof. Assume that $E=\bigcup_{i=0}^{\infty} E_{i}^{\prime}$ is any extension, $\delta_{i}$ is the default used in defining $E_{i}^{\prime}$, and the time bound is $n$. Then, we have

$$
E_{i}^{\prime}=T h\left(E_{i-1}^{\prime}\right) \bigcup \operatorname{Cons}\left(\left\{\delta_{i}\right\}\right)
$$

For the default

$$
\delta_{i}=\frac{\alpha_{i}: M \beta_{1}, \ldots, M \beta_{m}}{\omega_{i}}
$$

we easily have $E_{i-1}^{\prime} \vdash \alpha_{i}$, and $E \nvdash \neg \beta_{1}, \ldots, \neg \beta_{m}$.

From $\delta_{i}$ we can construct $D_{j}^{\prime}$ and $D_{j}^{\prime \prime}$ as follows:

$$
\begin{aligned}
& D_{j}^{\prime}=\left\{\begin{array}{cc}
\left\{\delta_{i}\right\} \quad \text { if } \omega_{i} \notin K_{j}, K_{j-1}, B_{j-1} \vdash_{n} \alpha_{i}, \text { and } \\
\quad \begin{array}{c}
K_{j-1}, B_{j-1} \nvdash_{n}^{F} \neg \beta_{l},(l=1, \ldots, m) . \\
\phi
\end{array} \quad \text { otherwise }
\end{array}\right.
\end{aligned}
$$

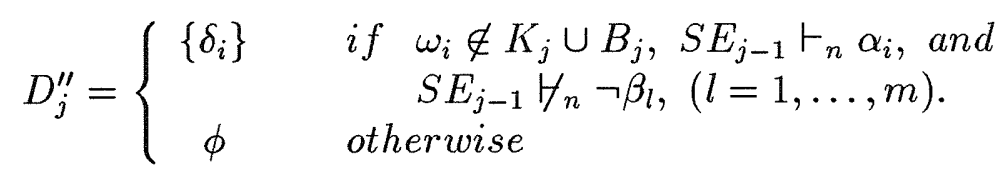

Obviously, $D_{j}^{\prime}$ and $D_{j}^{\prime \prime}$ satisfy Definition $4.1, D_{j}^{\prime} \cap D_{j}^{\prime \prime}=\phi$, and

$$
D_{j}^{\prime} \bigcup D_{j}^{\prime \prime}=\left\{\begin{array}{cc}
\left\{\delta_{i}\right\} & \text { if } \omega_{i} \notin K_{j}, S E_{j-1} \vdash_{n} \alpha_{i}, \text { and } \\
& \begin{array}{c}
S E_{j-1} \nvdash_{n} \neg \beta_{l},(l=1, \ldots, m) . \\
\text { otherwise }
\end{array}
\end{array}\right.
$$

(1) We prove the sequence of $S E_{1}, S E_{2}, \cdots$ can be constructed by using the defaults in the same order as in the construction of $E_{1}^{\prime}, E_{2}^{\prime}, \cdots$

(Base step):

Consider $E_{1}^{\prime}$ and $\delta_{1}$. From the assumption, we have $\alpha_{1} \in T h(W)$, that is, there exists a number $N_{1}$ such that $\alpha \in T h_{N_{1}}(W)$. Since

$$
T h_{N_{1}}(W)=\underbrace{T h_{n}\left(T h_{n}\left(\cdots\left(T h_{n}(W) \cdots\right)\right)\right)}_{\left\lceil\frac{N_{1}}{n}\right\rceil}=T h_{n}\left(K_{\left\lceil\frac{N_{1}}{n}\right\rceil-1}\right)
$$

and $K_{\left\lceil\frac{N_{1}}{n}\right\rceil-1} \subseteq S E_{\left\lceil\frac{N_{1}}{n}\right\rceil-1}$, we have $S E_{\left\lceil\frac{N_{1}}{n}\right\rceil-1} \vdash_{n} \alpha_{1}$. At the same time, since $E \forall \neg \beta_{l}$ and $S E_{\left\lceil\frac{N_{1}}{n}\right\rceil-1} \subseteq E$, we have $S E_{\left\lceil\frac{N_{1}}{n}\right\rceil-1} \nvdash_{n}{ }_{n} \neg \beta_{l}(l=1,2, \cdots, m)$. Therefore, exactly one of $D_{\left\lceil\frac{N_{1}}{n}\right\rceil-1}^{\prime}$ and $D_{\left\lceil\frac{N_{1}}{n}\right\rceil-1}^{\prime \prime}$ is $\left\{\delta_{1}\right\}$, that is, $\omega_{1} \in S E_{\left\lceil\frac{N_{1}}{n}\right\rceil}$ if $\omega_{1} \in E_{1}^{\prime}$. 


$$
\begin{aligned}
& K_{0} \quad=W \quad B_{0} \quad=\phi \\
& K_{1}=T h_{n}\left(K_{0}\right) \quad B_{1} \quad=T h_{n}^{\prime}\left(K_{0} \cup B_{0}\right) \\
& K_{\left\lceil\frac{N_{1}}{n}\right\rceil}=T h_{n}\left(K_{\left\lceil\frac{N_{1}}{n}\right\rceil-1}\right) \quad B_{\left\lceil\frac{N_{1}}{n}\right\rceil}=T h_{n}^{\prime}\left(K_{\left\lceil\frac{N_{1}}{n}\right\rceil-1} \cup B_{\left\lceil\frac{N_{1}}{n}\right\rceil-1}\right) \cup \operatorname{Cons}\left(D_{\left\lceil\frac{N_{1}}{n}\right\rceil-1}^{\prime}\right) \\
& K_{\left\lceil\frac{N_{1}}{n}\right\rceil+1}=T h_{n}\left(K_{\left\lceil\frac{N_{1}}{n}\right\rceil}\right) \quad B_{\left\lceil\frac{N_{1}}{n}\right\rceil+1}=T h_{n}^{\prime}\left(K_{\left\lceil\frac{N_{1}}{n}\right\rceil} \cup B_{\left\lceil\frac{N_{1}}{n}\right\rceil}\right) \\
& G_{0} \quad=\phi \\
& G_{1} \quad=T h_{n}^{\prime}\left(K_{0} \cup B_{0} \cup G_{0}\right) \\
& \vdots \\
& G_{\left\lceil\frac{N_{1}}{n}\right\rceil}=T h_{n}^{\prime}\left(K_{\left\lceil\frac{N_{1}}{n}\right\rceil-1} \cup B_{\left\lceil\frac{N_{1}}{n}\right\rceil-1} \cup G_{\left\lceil\frac{N_{1}}{n}\right\rceil-1}\right) \cup \operatorname{Cons}\left(D_{\left\lceil\frac{N_{1}}{n}\right\rceil-1}^{\prime \prime}\right) \\
& G_{\left\lceil\frac{N_{1}}{n}\right\rceil+1}=T h_{n}^{\prime}\left(K_{\left\lceil\frac{N_{1}}{n}\right\rceil} \cup B_{\left\lceil\frac{N_{1}}{n}\right\rceil} \cup B_{\left\lceil\frac{N_{1}}{n}\right\rceil}\right)
\end{aligned}
$$

Figure 1: The construction of the sequence of $S E_{1}, S E_{2}, \cdots$

\section{(Induction step)}

Assume $\omega_{i} \in S E_{j_{i}}$ for $\omega_{i} \in E_{i}^{\prime}(i \leq k)$. Then, we can show that $\omega_{k+1} \in S E_{j_{k+1}}$ if $\delta_{i+1}$ is used in $E_{i+1}^{\prime}$ and $\omega_{k+1} \in E_{k+1}^{\prime}$.

From $\omega_{k+1} \in E_{k+1}^{\prime}$, we have $E_{k}^{\prime} \vdash \alpha_{k+1}$ and $E \nvdash \neg \beta_{1}, \ldots, \neg \beta_{m}$. If $E_{k}^{\prime} \vdash \alpha_{k+1}$, there exists a number $N_{k+1}$ such that $\alpha_{k+1} \in T h_{N_{k+1}}\left(W \cup\left\{\omega_{1}, \cdots, \omega_{k}\right\}\right)$. By the assumption of induction and the monotonicity of $S E_{j}$, we have $\left(W \cup\left\{\omega_{1}, \cdots, \omega_{k}\right\}\right) \subseteq S E_{j_{k}}$. Then, by the same discussions as in the base step, we can prove $\omega_{k+1} \in S E_{j_{k+1}}$. Therefore, $\omega_{i} \in A E$ if $\omega_{i} \in E$. That is,

$$
A E=\bigcup_{j=0}^{\infty} S E_{j} \subseteq \bigcup_{i=0}^{\infty} E_{i}^{\prime}=E .
$$

Such a construction of the sequence $S E_{1}, S E_{2}, \ldots, S E_{\left\lceil\frac{N_{1}}{n}\right\rceil}, \ldots$ is depicted in Figure 1.

(2) Furthermore, we show that any closed wff of $E$ is in some $S E_{j}$.

The closed wff in an extension can be divided into those derived from defaults and those derived from Th-operator. Since $\alpha \in E$ and $E=\bigcup_{i=0}^{\infty} E_{i}^{\prime}$, there exists a $k$ such that $\alpha \in E_{k}^{\prime}$. Hence, $\alpha \in T h\left(W \cup\left\{\omega_{1}, \omega_{2}, \cdots, \omega_{k}\right\}\right)$. Then, there exists an $N$ such that $\left(W \cup\left\{\omega_{1}, \omega_{2}, \cdots, \omega_{k}\right\}\right) \vdash_{N} \alpha$. On the other hand, the above construction method guarantees that there exists a $j$ such that $\left(W \cup\left\{\omega_{1}, \omega_{2}, \cdots, \omega_{k}\right\}\right) \subseteq S E_{j}$. Therefore, we 
have

$$
\alpha \in \underbrace{T h_{n}\left(T h_{n}\left(\cdots\left(T h_{n}\left(S E_{j}\right) \cdots\right)\right)\right)}_{\left\lceil\frac{N}{n}\right\rceil} \subseteq A E,
$$

which means

$$
A E=\bigcup_{j=0}^{\infty} S E_{j} \supseteq \bigcup_{i=0}^{\infty} E_{i}^{\prime}=E
$$

From (1) and (2) above, we have $A E=E$.

Corollary 4.4 Let $\mathbf{E}$ be the set of all extensions of a closed default theory $\Delta$, and $\mathbf{A E}$ be the set of all approximate extensions of $\Delta$. Then, $\mathbf{E} \subseteq \mathbf{A E}$.

\section{Some examples}

In this section, we give some examples to illustrate the construction procedure of subextensions and approximate extensions.

Example 5.1 Let $\Delta_{1}=(D, W)$ be a default theory with

$$
\begin{aligned}
D & =\left\{\delta_{1}=\frac{: M A}{\neg B}, \quad \delta_{2}=\frac{: M B}{\neg C}, \quad \delta_{3}=\frac{: M C}{\neg A}\right\}, \\
W & =\phi
\end{aligned}
$$

and a time-bound be 3 .

Though there are no extensions for $\Delta_{1}$, we can successively construct seven approximate extensions from $\Delta_{1}$ by Definition 4.1 and 4.2 as shown in Table 1 .

\begin{tabular}{|l||l|l|l|l|l|}
\hline & $S E_{0}^{(i)}$ & $S E_{1}^{(i)}$ & $S E_{2}^{(i)}$ & $S E_{3}^{(i)}$ & $S E_{4}^{(i)}$ \\
\hline \hline$A E^{(1)}$ & $\phi$ & $\phi$ & & & \\
\hline$A E^{(2)}$ & $\phi$ & $\{\neg B\}$ & $\{\neg B\}$ & & \\
\hline$A E^{(3)}$ & $\phi$ & $\{\neg B\}$ & $\{\neg B, \neg A\}$ & $\{\neg B, \neg A\}$ & \\
\hline$A E^{(4)}$ & $\phi$ & $\{\neg C\}$ & $\{\neg C\}$ & & \\
\hline$A E^{(5)}$ & $\phi$ & $\{\neg C\}$ & $\{\neg C, \neg B\}$ & $\{\neg C, \neg B\}$ & \\
\hline$A E^{(6)}$ & $\phi$ & $\{\neg A\}$ & $\{\neg A\}$ & & \\
\hline$A E^{(7)}$ & $\phi$ & $\{\neg A\}$ & $\{\neg A, \neg C\}$ & $\{\neg A, \neg C\}$ & \\
\hline
\end{tabular}

Table 1: The approximate extensions of $\Delta_{1}$

Example 5.2 Let $\Delta_{2}=(D, W)$ be a default theory with

$$
\begin{aligned}
& D=\left\{\delta_{1}=\frac{: M A}{\neg B}, \delta_{2}=\frac{: M B}{\neg C}, \delta_{3}=\frac{: M C}{\neg D}, \delta_{3}=\frac{: M D}{\neg A}\right\}, \\
& W=\phi,
\end{aligned}
$$


and a time-bound be 3 . There are two extensions $\{\neg B, \neg D\}$ and $\{\neg A, \neg C\}$ for $\Delta_{2}$. On the other hand, we can construct the following nine approximate extensions from $\Delta_{2}$ by Definition 4.1 and 4.2 as shown in Table 2. Obviously, we have $A E^{(3)}=A E^{(7)}$ and $A E^{(5)}=A E^{(9)}$ that are all the extensions of $\Delta_{2}$.

\begin{tabular}{|l||l|l|l|l|l|}
\hline & $S E_{0}^{(i)}$ & $S E_{1}^{(i)}$ & $S E_{2}^{(i)}$ & $S E_{3}^{(i)}$ & $S E_{4}^{(i)}$ \\
\hline \hline$A E^{(1)}$ & $\phi$ & $\phi$ & & & \\
\hline$A E^{(2)}$ & $\phi$ & $\{\neg B\}$ & $\{\neg B\}$ & & \\
\hline$A E^{(3)}$ & $\phi$ & $\{\neg B\}$ & $\{\neg B, \neg D\}$ & $\{\neg B, \neg D\}$ & \\
\hline$A E^{(4)}$ & $\phi$ & $\{\neg C\}$ & $\{\neg C\}$ & & \\
\hline$A E^{(5)}$ & $\phi$ & $\{\neg C\}$ & $\{\neg C, \neg A\}$ & $\{\neg C, \neg A\}$ & \\
\hline$A E^{(6)}$ & $\phi$ & $\{\neg D\}$ & $\{\neg D\}$ & & \\
\hline$A E^{(7)}$ & $\phi$ & $\{\neg D\}$ & $\{\neg D, \neg B\}$ & $\{\neg D, \neg B\}$ & \\
\hline$A E^{(8)}$ & $\phi$ & $\{\neg A\}$ & $\{\neg A\}$ & & \\
\hline$A E^{(9)}$ & $\phi$ & $\{\neg A\}$ & $\{\neg A, \neg C\}$ & $\{\neg A, \neg C\}$ & \\
\hline
\end{tabular}

Table 2: The approximate extensions of $\Delta_{2}$

Furthermore, we can show that $\{\neg A, \neg B, \neg C, \neg D\},\{\neg A, \neg B, \neg C\}$, and $\{\neg A, \neg B, \neg D\}$ are pseudo extensions of the default theory $\Delta_{2}$ (Yuasa[8]), but they are not the approximate extensions. The converse is not true. In fact, $A E^{(1)}$ is not a pseudo extension. Hence, from this example, we have $\mathbf{F} \nsubseteq \mathbf{A E}$ and $\mathbf{A E} \nsubseteq \mathbf{F}$.

Example 5.3 Let $\Delta_{3}=(D, W)$ be a default theory with

$$
\begin{aligned}
& D=\left\{\frac{\operatorname{student}(X): M \neg \text { married }(X)}{\neg \text { married }(X)}, \frac{\operatorname{adult}(X): M \operatorname{married}(X)}{\operatorname{married}(X)}\right\}, \\
& W=\{\operatorname{adult}(X) \leftarrow \operatorname{student}(X) ., \quad \operatorname{student}(B o b) .\},
\end{aligned}
$$

and a time-bound be 3 . There are two extensions $E^{(1)}$ and $E^{(2)}$ for $\Delta_{3}$ as shown in Table 3 .

\begin{tabular}{|l|l|}
\hline \multicolumn{1}{|c|}{$E^{(1)}$} & \multicolumn{1}{|c|}{$E^{(2)}$} \\
\hline \hline adult $(B o b) \leftarrow$ student $(B o b) .$, & adult $(B o b) \leftarrow$ student $(B o b) .$, \\
student $(B o b)$, adult $(B o b)$, & student $(B o b)$, adult $(B o b)$, \\
$\neg$ married $(B o b)$ & married $(B o b)$ \\
\hline
\end{tabular}

Table 3: The extensions of $\Delta_{3}$

We can successively construct three approximate extensions as in Table 4. 


\begin{tabular}{|c|c|c|c|}
\hline & $S E_{0}^{(i)}$ & $S E_{1}^{(i)}$ & $S E_{2}^{(i)}$ \\
\hline$A E^{(1)}$ & $\begin{array}{l}\text { adult }(B o b) \leftarrow \text { student }(B o b) . \\
\text { student }(B o b)\end{array}$ & $\begin{array}{l}\text { adult }(B o b) \leftarrow \text { student }(B \circ b) . \\
\text { student }(B o b), \text { adult }(B o b)\end{array}$ & $\begin{array}{l}\text { adult }(B o b) \leftarrow \text { student }(B o b) . \\
\text { student }(B o b), \text { adult }(B \circ b)\end{array}$ \\
\hline$A E^{(2)}$ & $\begin{array}{l}\text { adult }(B o b) \leftarrow \text { student }(B o b) . \\
\text { student }(B o b)\end{array}$ & $\begin{array}{l}\text { adult }(B o b) \leftarrow \text { student }(B o b) \text {. } \\
\text { student }(B o b), \text { adult }(B o b) \\
\neg \text { married }(B o b)\end{array}$ & $\begin{array}{l}\text { adult }(B o b) \leftarrow \text { student }(B \circ b) \text {. } \\
\text { student }(B o b), \text { adult }(B \circ b) \\
\neg \text { married }(B o b)\end{array}$ \\
\hline$A E^{(3)}$ & $\begin{array}{l}\text { adult }(B o b) \leftarrow \text { student }(B o b) . \\
\text { student }(B o b)\end{array}$ & $\begin{array}{l}\text { adult }(B o b) \leftarrow \text { student }(B o b) \text {. } \\
\text { student }(B o b), \text { adult }(B \circ b) \\
\text { married }(B o b)\end{array}$ & $\begin{array}{l}\text { adult }(B o b) \leftarrow \text { student }(B o b) \text {. } \\
\text { student }(B o b), \text { adult }(B o b) \\
\text { married }(B o b)\end{array}$ \\
\hline
\end{tabular}

Table 4: The approximate extensions of $\Delta_{3}$

$A E^{(1)}$ is a minimal approximate extension which is equal to $T h(W) . A E^{(2)}$ and $A E^{(3)}$ are the same as the extensions $E^{(1)}$ and $E^{(2)}$, respectively.

Example 5.4 Let $\Delta_{4}=(D, W)$ be a default theory with

$$
\begin{aligned}
& D=\left\{\frac{\operatorname{num}(X): M \neg \operatorname{even}(X)}{\operatorname{odd}(X)}\right\} \\
& W=\{\operatorname{num}(s(X)) \leftarrow \operatorname{num}(X) ., \quad \text { even }(s(s(X)) \leftarrow \operatorname{even}(X) ., \text { even }(0) ., \quad \text { num }(s(0))\}
\end{aligned}
$$

and a time-bound be 3 . This default theory has a unique extension $E$ :

$$
E=T h\left(W \cup\left\{\operatorname{odd}(s(0)), \quad \operatorname{odd}\left(s^{3}(0)\right), \quad \operatorname{odd}\left(s^{5}(0)\right), \cdots, \quad \operatorname{odd}\left(s^{2} j(s(0))\right), \cdots\right\}\right) .
$$

Since this default theory is infinite, there are infinitely many possible approximate extensions. Here we just show the part of approximate extension $A E^{(i)}$.

$$
\begin{aligned}
& S E_{1}^{(i)}=\left\{\begin{array}{l}
\operatorname{num}(s(0)), \quad \operatorname{num}\left(s^{2}(0)\right), \quad \operatorname{num}\left(s^{3}(0)\right), \quad \operatorname{num}\left(s^{4}(0)\right) \\
\operatorname{even}(0), \quad \operatorname{even}\left(s^{2}(0)\right), \quad \text { even }\left(s^{4}(0)\right), \quad \text { even }\left(s^{6}(0)\right) \\
\operatorname{odd}(s(0))
\end{array}\right\} \\
& S E_{2}^{(i)}=\left\{\begin{array}{l}
\operatorname{num}(s(0)), \quad \operatorname{num}\left(s^{2}(0)\right), \quad \operatorname{num}\left(s^{3}(0)\right), \quad \operatorname{num}\left(s^{4}(0)\right) \\
\operatorname{num}\left(s^{5}(0)\right), \quad \operatorname{num}\left(s^{6}(0)\right), \quad \operatorname{num}\left(s^{7}(0)\right), \ldots \\
\operatorname{even}(0), \quad \operatorname{even}\left(s^{2}(0)\right), \quad \operatorname{even}\left(s^{4}(0)\right), \quad \operatorname{even}\left(s^{6}(0)\right) \\
\ldots \\
\operatorname{odd}(s(0)), \quad \operatorname{odd}\left(s^{3}(0)\right)
\end{array}\right\} \\
& S E_{3}^{(i)}=\ldots
\end{aligned}
$$

From these examples above, we can see the following: all approximate extensions have $T h(W)$ as its knowledge part $K\left(=\bigcup_{i=0}^{\infty} K_{i}\right)$. However, the belief part $B\left(=\bigcup_{i=0}^{\infty} B_{i}\right)$ and the guess part $G\left(=\bigcup_{i=0}^{\infty} G_{i}\right)$ in an approximate extension depends on the order of selected defaults. 


\section{Concluding remarks}

Reiter's default theories has a difficulty that the extensions can not be constructed successively. Since the extensions should be candidates of possible belief sets, it is important to construct the extensions. Many efforts to attack this problem have been made by many researchers. For example, Murakami et al. [2], and Yuasa et al. [9] have provided two new kinds of non-recursive extension and pseudo extension which can successively be constructed, and showed that the pseudo extensions include the extensions. However, their methods are still not computable. Particularly, the termination of every step of construction is not guaranteed in the first order default logic.

As a solution to such a problem, we have introduced the notions of sub-extensions and approximate extensions which can successively be constructed and every construction step terminates in a finite time. Furthermore, our approximate extensions include Reiter's extension.

In the first order default reasoning systems, there are another two problems which should be addressed: (a) An infinite extension of a default theory. (b) The termination problem of derivation in the first order logic. We can not have an infinite amount of time to compute an infinite extension. What we can do is just to compute approximately some finite parts of extensions by using the time-bounded reasoning. Such finite parts are useful and important, since they approximately represent a part of possible belief sets in some way. Our sub-extensions are just such finite parts. Classifying $A E$, we can infer the results more efficiently within the time-bound.

Furthermore, the notion of time-bounded reasoning will change many concepts such as consistency, beliefs, reliability, queries, and so on. In another paper [6], we will discuss these concepts and show the notions of sub-extension and approximate extension help in the default knowledge base systems.

\section{References}

[1] K. Murakami. Default reasoning under restricted number of reasoning. Trans. Informaion Processing Society Japan, Vol. 32, pp. 364-372, 1991. (in Japanese).

[2] K. Murakami, T. Aibara, and H. Shitanda. Nonrecursive extension and its properties in default reasoning. Journal Japanese Society for Artificial Intelligence, Vol. 3, pp. 359367, 1988. (in Japanese).

[3] R. Reiter. A logic for default reasoning. Journal of Artifical Intelligence, Vol. 13, No. 1-2, pp. 81-132, April 1980. 
[4] R. Reiter and G. Criscuolo. On interacting defaults. In Proceedings of the 7th International Joint Conference on Artificial Intelligence, pp. 270-276, 1981.

[5] Y. Shi. The principles of time-bounded knowledge base management systems. Engineering Sciences Reports, Kyushu University, Vol. 11, No. 1, pp. 77-84, 1989. (in Japanese).

[6] Y. Shi and S. Arikawa. Sub-extensions in the default knowledge base systems. to appear, 1991.

[7] Y. Shi and S. Arikawa. Time-bounded reasoning in first order knowledge base systems. Lecture Notes in Artificial Intelligence, Vol. 485, pp. 54-72, 1991.

[8] H. Yuasa. Pseudo extensions in default reasoning and belief revision using model inference. Master's thesis, Dept. Information Systems, Kyushu University, 1988. (in Japanese).

[9] H. Yuasa and S. Arikawa. Pseudo extension in default reasoning and belief revision by model inference. Lecture Notes in Artificial Intelligence, Vol. 383, pp. 27-37, 1989. 


\section{About the Authors}

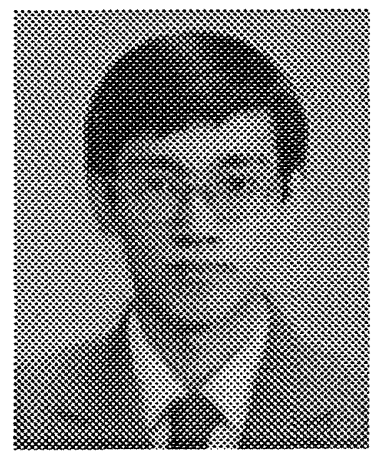

Yihua Shi (史 一華) was born in Jiansu Province of China on January 14, 1961. He received the B.S. degree from Fudan University of China in 1982 and the M.S. degree from Kyushu University in 1989. Presently, he is a graduate student of Doctor Course in Information Systems, Kyushu University. His interests are in truth maintenance, reasoning and nonmonotonic logic.

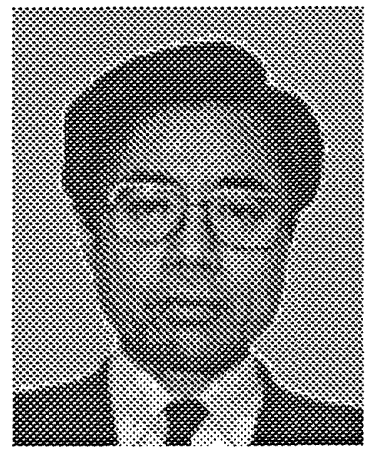

Setsuo Arikawa (有川 節夫) was born in Kagoshima on April 29,1941 . He received the B.S. degree in 1964, the M.S. degree in 1966 and the Dr.Sci. degree in 1969 all in Mathematics from Kyushu University. Presently, he is Professor of Research Institute of Fundamental Inforamtion Science, Kyushu University. His research interests include algorithmic learning theory, logic and inference in $\mathrm{AI}$, and information retrieval systems. 